Brit. Heart f., 1967, 29, 299.

\title{
Left Atrial Impulse Formation in Atrial Flutter
}

\author{
M. MIROWSKI AND W. J. ALKAN \\ From the Division of Cardiology and the Department of Medicine "A", Asaf Harofe Government Hospital, \\ Zrifin, Israel
}

Although the underlying mechanism of atrial flutter has not yet been conclusively determined (Katz and Pick, 1960), the available evidence increasingly favours the "unifocal" versus the "circus movement" theory (Bellet, 1963). While this arrhythmia may be produced experimentally by an appropriate stimulation of any atrial area (Scherf, Blumenfeld, and Yildiz, 1963), the precise location of the discharging focus in clinical instances of flutter remains hypothetical. Data pertaining to this problem derive essentially from theoretical considerations based on the direction of $\mathbf{P}$ waves as recorded in the limb and osophageal leads. Thus, it is held that in the "common type" of flutter, characterized by inverted $P$ waves in leads II, III, and aVF, the impulses originate in the caudal region of the atria (Prinzmetal et al., 1952), most probably in the atrio-ventricular (A-V) node (Scherf and Cohen, 1964; Puech, 1956; Marques, Mota, and Nogueira, 1962). On the other hand, in the "uncommon type" of flutter, in which the $P$ waves are upright in leads II, III, and aVF, a cephalad origin of impulses has been postulated (Prinzmetal et al., 1952).

The value of conclusions obtained in this way is obviously limited to information deduced only from the direction of the vertical component of the atrial vector. The present study attempts to overcome this limitation by approaching the atrial electrical activity as a tridimensional phenomenon. This aim is achieved by analysing the atrial deflections vectorially in the præcordial leads in addition to the limb leads. Since the axes of the præcordial leads define approximately the horizontal plane of the body (Massie and Walsh, 1960), the direction of the horizontal $\mathbf{P}$ axis is easily determined, making available essential information concerning the sagittal

Received March 3, 1966. and transverse components of the dipole vector . Knowing the directions of its three perpendicular components, the spatial orientation of the mean atrial vector is subsequently inferred. It is assumed that the negative extremity of this vector indicates, with reasonable accuracy, the site of the initiating focus.

This approach, when recently applied to ectopic atrial rhythms of normal and regular rate (Mirowski, Neill, and Taussig, 1963; Mirowski, 1966a, c), led to the description of several hitherto unknown patterns of left atrial rhythm and provided means for differentiation of this arrhythmia from those arising in the region of the A-V node. Application of similar principles of analysis to atrial flutter and tachycardia was a logical corollary to these studies (Mirowski, 1966b). It soon became apparent that left atrial impulse formation, a mechanism not previously considered, is of importance in the genesis of atrial flutter. In the present communication we intend to discuss the possible role of left atrial automaticity in this arrhythmia and to report four examples in which a posteriorly located left atrial pacemaker is demonstrated. In all these instances it is of particular interest that the atrial deflections in lead V1 exhibit a distinctive "dome and dart" configuration (Fig. 1), previously reported as characteristic of left atrial rhythm (Mirowski et al., 1963; Mirowski, 1966a).

\section{Clinical and Electrocardiographic Data}

Case 1. A 74-year-old man was admitted in congestive heart failure due to arteriosclerotic heart disease. The electrocardiogram (Fig. 2) showed atrial flutter of $260 / \mathrm{min}$. with irregular ventricular response. $P$ waves were inverted in leads I, II, III, aVF, V5, and V6. In lead V1 a distinctive "dome and dart" configuration of the $P$ waves was noted (Fig. 2 and 3 ). The frontal and

* The horizontal lead reference frame employed for this purpose has been described elsewhere (Mirowski, 1966a). 


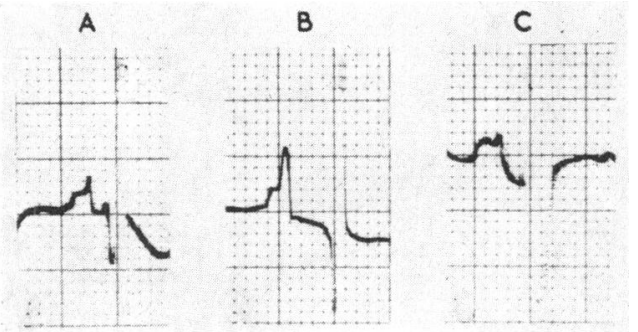

Fig. 1.-Typical "dome and dart" $P$ waves. A. No atrial enlargement. B. Right atrial enlargement. C. Left atrial enlargement. (Reproduced from Mirowski, Neill, and Taussig (1963), by permission of the American Heart Association, Inc.).

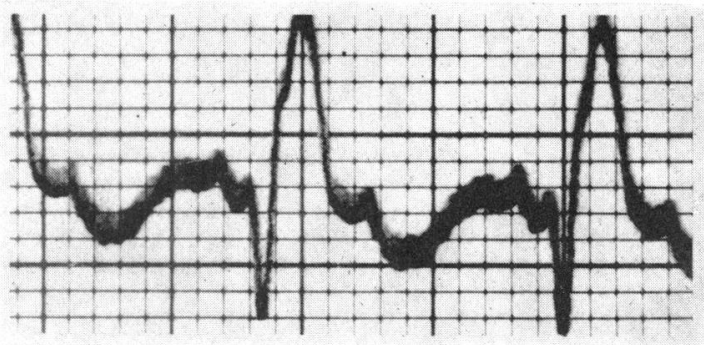

FIG. 3.-Case 1. Lead V1. Typical "dome and dart" $P$ waves (compare with Fig. 1A). Enlarged from a tracing recorded at a different time from that reproduced in Fig. 2. horizontal $P$ vectors were $-120^{\circ}$ and $+150^{\circ}$, respectively, with a mean spatial vector pointing upwards, anteriorly, and to the right.

Case 2. A 48-year-old man had rheumatic mitral stenosis and insufficiency. A chest $x$-ray film showed enlargement of the left atrium. The electrocardiogram (Fig. 4) revealed atrial flutter of $333 / \mathrm{min}$. with irregular ventricular response. With the exception of lead aVR, the $P$ waves were inverted in the extremity leads. In the chest leads, $P$ waves were inverted over the left præcordium and exhibited typical "dome and dart" configuration in lead V1 (Fig. 4 and 5), with the first component as high as the second, suggestive of left atrial hypertrophy. The frontal and horizontal $P$ axes were approximately $-130^{\circ}$ and $+150^{\circ}$, respectively, and the spatial mean $\mathbf{P}$ vector pointed upwards, anteriorly, and to the right.

Case 3. A 40-year-old man with a history of mitral commissurotomy 11 years ago was referred to the Asaf Harofe Hospital for assessment of his cardiac condition. The electrocardiogram revealed atrial flutter of $330 / \mathrm{min}$. with a $2: 1$ block. The atrial complexes were upright in

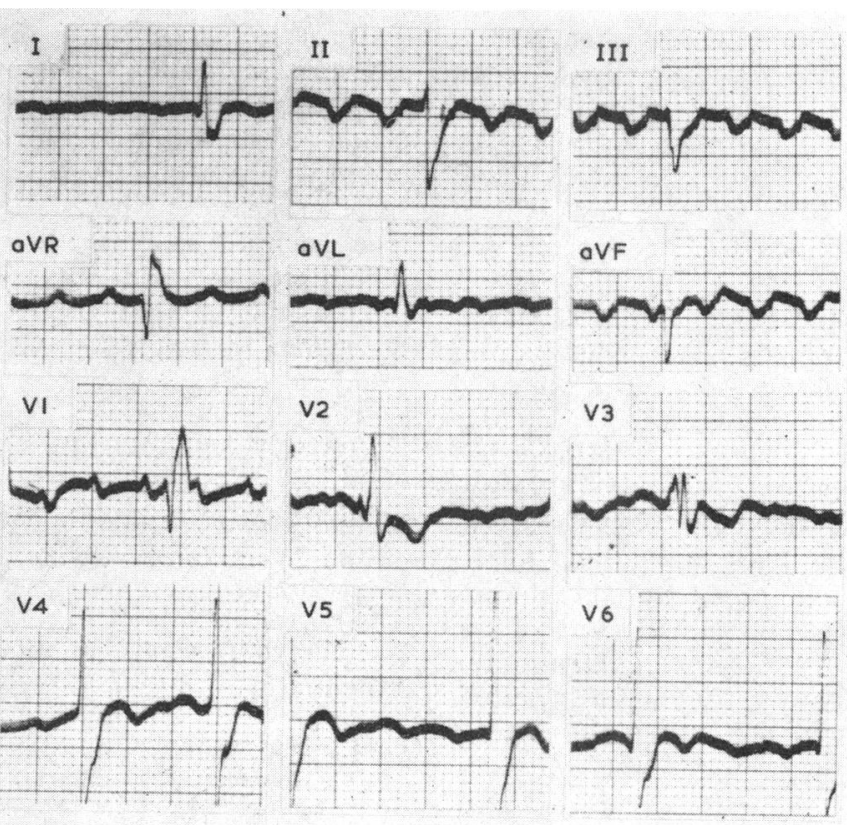

FIG. 2.-Case 1. Atrial flutter. Inverted P waves in lead I and V6, "dome and dart" in lead V1. 

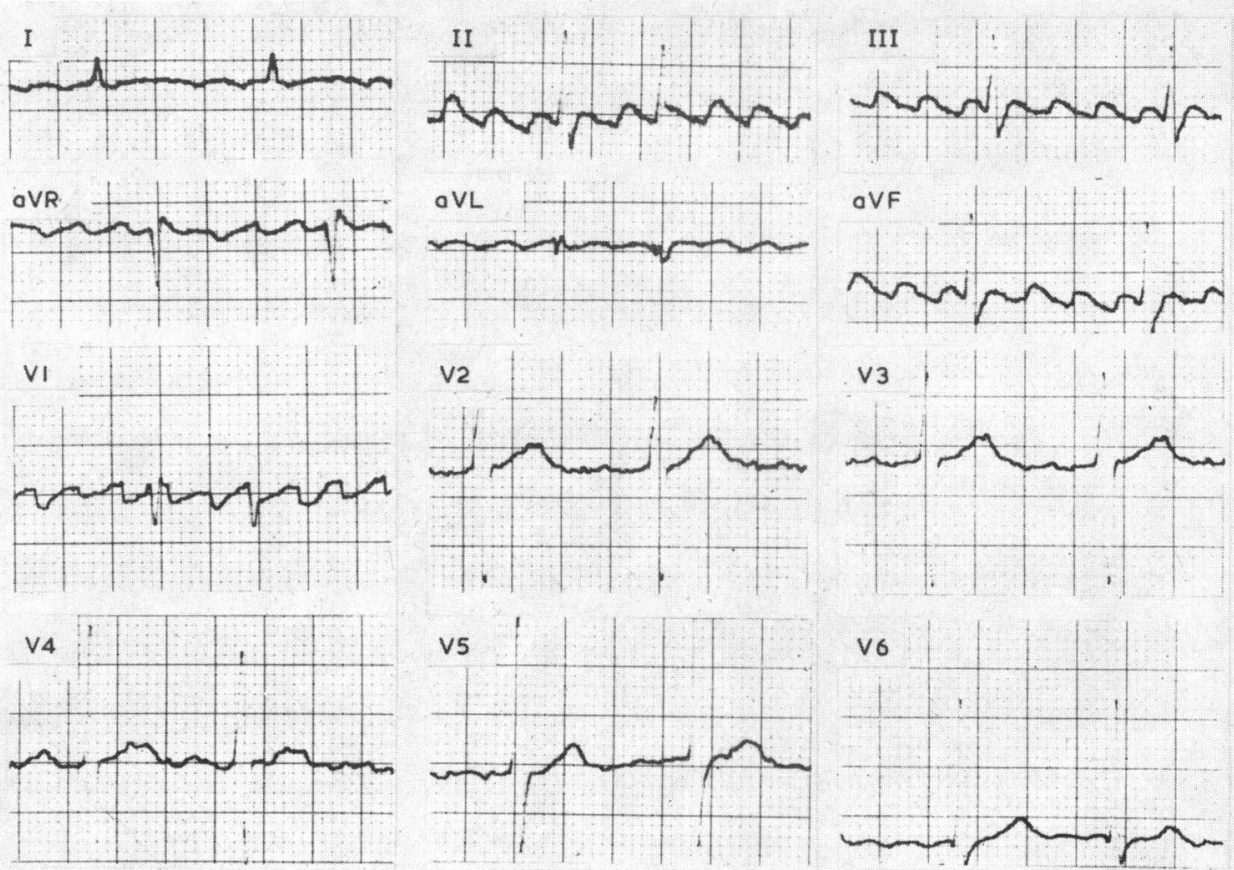

FIg. 4.-Case 2. Atrial flutter. Inverted $P$ waves in leads I and V6. Typical "dome and dart" $P$ waves in lead V1.

leads II, III, and aVF, isoelectric in lead I, and inverted in aVL $\left(\hat{A P}:+90^{\circ}\right)$. In the præcordial leads the $P$ waves were inverted in lead V6 and upright in V1 where they exhibited a typical "dome and dart" configuration (Fig. 6). The spatial $P$ vector pointed downwards, anteriorly, and to the right.

Case 4. A 67-year-old man was admitted for pneumonia and mild congestive heart failure. The electrocardiogram (Fig. 7) revealed atrial flutter of $375 / \mathrm{min}$. with irregular ventricular response. The atrial deflections were inverted in leads I and aVL, upright in II, III, aVR, and aVF (ÂP: $+135^{\circ}$ ). In the præcordial leads $P$ waves were upright in V1-V3 and negative in

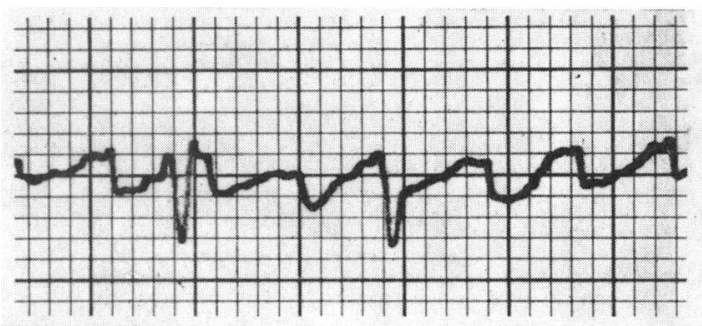

FIG. 5.-Case 2. Lead V1 (enlarged from Fig. 4). Details of "dome and dart" $P$ waves. The breadth of the first component suggests left atrial hypertrophy (compare with Fig. 1C).

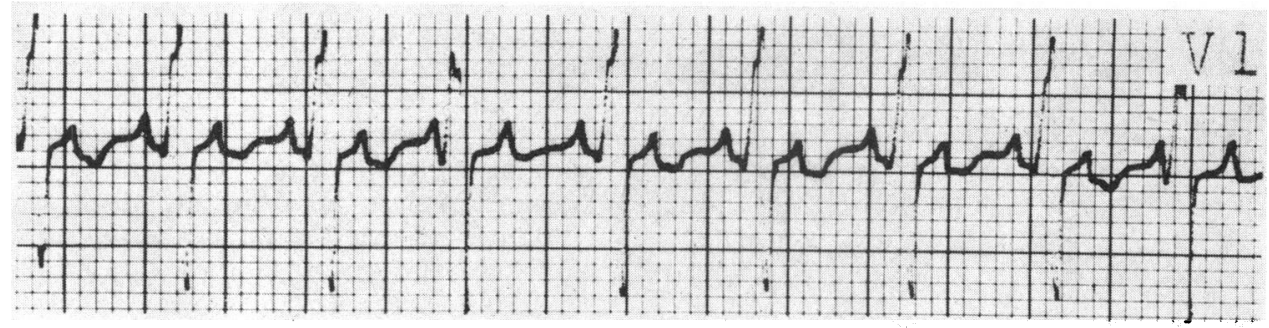

FIG. 6.-Case 3. Lead V1. Typical "dome and dart" $P$ waves. 


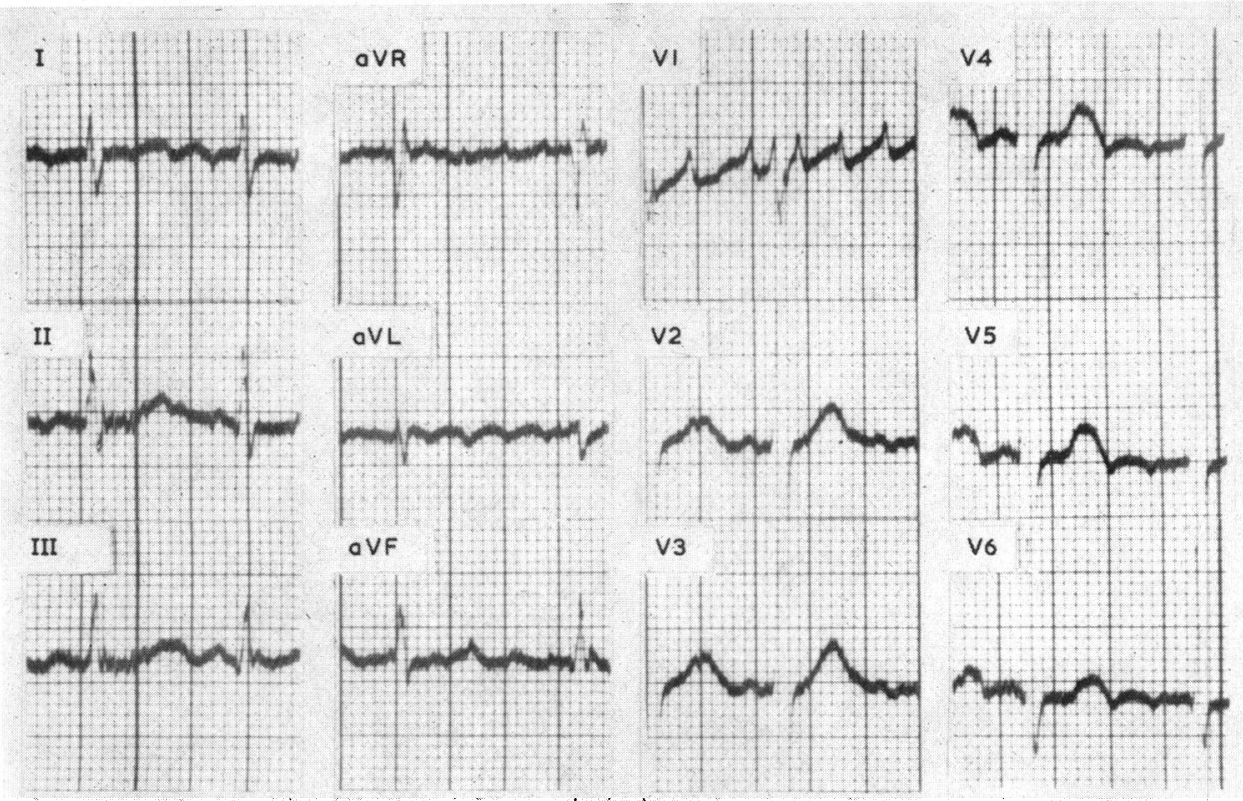

FIG. 7.-Case 4. Atrial flutter. Inverted $P$ waves in leads $I$ and V6, and "dome and dart" $P$ waves in lead V1. Leads I, II, and III; aVR, aVL, and aVF; V1, V2, and V3; V5 and V6 are recorded simultaneously.

V4-V6. In lead V1 the $P$ waves exhibited "dome and dart" configuration, with the "dart" component of $3 \mathrm{~mm}$. amplitude (Fig. 7 and 8 ). The horizontal $P$ axis was $+140^{\circ}$ and the spatial atrial vector pointed downward, anteriorly and to the right.

\section{Discussion}

Vectorial analysis demonstrates that in our four patients with atrial flutter the pacemaker is located posteriorly in the left atrium. This conclusion is supported by a considerable chain of evidence.

The first link in this chain is represented by inverted $P$ waves in lead $I$. This finding indicates, with some rare exceptions (Mirowski and Vure, 1966), a left-to-right direction of the mean $P$ vector, and is consistent, in the absence of atrial inversion,

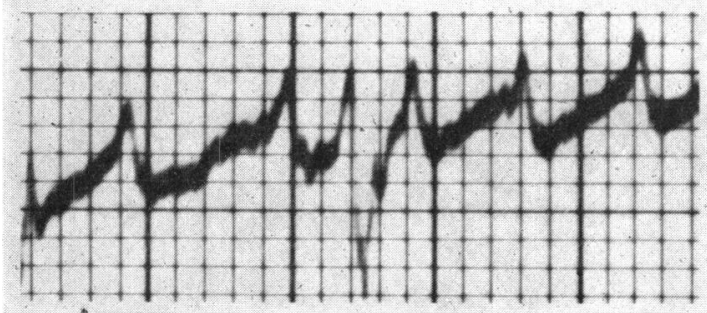

FIG. 8.-Case 4. Lead V1 (enlarged from Fig. 7). Details of the "dome and dart" $P$ waves. Note the conspicuous "dart" component (compare with Fig. 1B). with a spread of the activation wave from the left to the right atrium.

Additional support is provided by negative $P$ waves in lead V6 in all our cases. Although, theoretically, leads I and V6 are similar in their response to the transverse component of the dipole vector, it has recently been shown that the polarity of $\mathbf{P}$ waves in lead V6 reflects the course of atrial activation along the transverse axis more faithfully than it does in lead I (Mirowski, 1966a). Accordingly, negative $P$ waves in lead V6 have been described as the most sensitive and specific sign of left atrial impulse formation.

The distinctive configuration of the atrial deflection in lead V1 constitutes a third feature of considerable significance. These deflections are formed by an initial rounded, low-voltage component, followed by a sharp higher peak (Fig. 3, 5, 6 , and 8 ) and are similar to those previously described as the "dome and dart" $P$ waves (Fig. 1). Their importance derives from the fact that, when recorded in the semidirect right atrial leads, they are highly suggestive of a posteriorly located pacemaker in the left atrium.

Characteristic modifications in the configuration of "dome and dart" $P$ waves due to atrial hypertrophy are also observed in the cases presented. Left atrial enlargement was unequivocally demonstrated by $x$-ray examination in Case 2 and, in fact, 
the "dome" component was particularly prominent (Fig. 5). Case 4, which most probably had some degree of right atrial overloading due to acute respiratory distress, exhibited a conspicuous "dart" component (Fig. 8).

Finally, the direction of the $P$ vectors confirms our interpretation concerning the significance of the findings discussed above. In Cases 1 and 2 the spatial $P$ vector was found to be directed upwards, anteriorly, and to the right, indicating a posteroinferior site of impulse formation in the left atrium, while in Cases 3 and 4 this vector pointed downwards, anteriorly, and to the right, suggesting a postero-superior left atrial pacemaker.

Our observations confirm the diagnostic value of "dome and dart" $P$ waves as indicative of a posteriorly located ectopic focus in the left atrium, independent of its rate of discharge. The close resemblance between the salient electrocardiographic characteristics of the examples presented of atrial flutter and instances of left atrial rhythm of normal and regular rate with "dome and dart" $P$ waves reported previously (Mirowski et al., 1963; Mirowski, 1964, 1966a), indicates a similar sequence and path of spread of activation and implies a close proximity if not identity of the site of origin of the impulses. The possibility that we are dealing with the same pacemaker or group of pacemakers should be considered, since most arrhythmias result from changes in the firing rate of normally automatic specialized fibres (Hoffman and Cranefield, 1964).

The demonstration of left atrial automaticity in the above instances of atrial flutter suggests that such mechanisms may be frequent in the genesis of this arrhythmia in general. This is supported by the clinical observation that atrial flutter usually occurs in patients with mitral and arteriosclerotic heart disease in which the left atrium bears the burden of the hæmodynamic overload. On the other hand, atrial flutter is rare in normal hearts and in conditions in which the load is borne exclusively by the right atrium.

These considerations are further supported by the experimental work of Puech (1956) and of Hoffman and Cranefield (1964). Puech, while studying the sequence and direction of atrial activation in the common type of flutter by œsophageal and intracavitary leads, found activation of the two atria clearly dissociated, the left atrium being depolarized before the right one. Depolarization first progressed superiorly and then anteroinferiorly, the antero-inferior part of the right atrium being the last to be activated. Although these findings were interpreted as compatible with a focus of impulse formation in the A-V node (Puech, 1956; Scherf and Cohen, 1964), in our opinion they rather indicate a posterior left atrial pacemaker. Indeed, the anterior spread of activation, so clearly demonstrated in these experiments, is difficult to relate to an $A-V$ nodal origin when one recalls that the A-V node lies in the right atrium just behind the medial leaflet of the tricuspid valve and there is very little, if any, excitable atrial tissue anterior to this structure. Moreover, were the pacemaker located in the A-V node, one would expect, in addition to posterior spread of depolarization, initial activation of the right atrium or at least simultaneous activation of both rather than initial activation of the left atrium as demonstrated by Puech. While initial left atrial activation has been reported to occur in experimental A-V nodal rhythms (Brumlik, 1958; Scherf and Cohen, 1964), these observations will probably have to be re-evaluated in the light of more recent studies which demonstrate that the current criteria for the diagnosis of $A-V$ nodal rhythms are inadequate and that, in fact, many of the so-called "nodal" rhythms originate in the left atrium (Mirowski, 1966a, c).

An important contribution was recently made by Hoffman and Cranefield (1964), who investigated the distribution of automatic cells in the atria and concluded that there were no such cells in the A-V node. Since, in these authors' opinion, the presence of automatic cells represents the sole criterion for a given area of the heart to act as a pacemaker, the very automaticity of the $A-V$ node is now being seriously questioned. The fact that Hoffman and Cranefield suggest the existence of automatic cells at the junction of the pulmonary veins with the left atrium is of significance as, if it were so, it would provide an anatomo-physiological basis for the concept of left atrial arrhythmias.

\section{SUMMARY}

Four cases of atrial flutter of left atrial origin are reported. The electrocardiograms were characterized by distinctive "dome and dart" $P$ waves in lead V1 and inverted $P$ waves in leads I and V6. Vectorial analysis of atrial activation located the ectopic focus in the posterior portion of the left atrium.

The possible role of left atrial automaticity in the genesis of atrial flutter is discussed.

\section{REFERENCES}

Bellet, S. (1963). Clinical Disorders of the Heart Beat, 2nd ed., p. 140. Lea and Febiger, Philadelphia.

Brumlik, J. V. (1958). The sinoatrial node, the atrioventricular node and atrial dysrhythmias. In Advances in Electrocardiography, ed. C. E. Kossmann, p. 252. Grune and Stratton, New York and London. 
Hoffman, B. F., and Cranefield, P. F. (1964). The physiological basis of cardiac arrhythmias. Amer. F. Med., 37, 670.

Katz, L. N., and Pick, A. (1960). Current status of theories of mechanisms of atrial tachycardias, flutter and fibrillation. Progr. cardiovasc. Dis., 2, 651.

Marques, M. G., Mota, J. C., and Nogueira, R. A. (1962). The mechanism of atrial flutter. Cardiologia (Basel), 40, 269.

Massie, E., and Walsh, T. J. (1960). Clinical Vectorcardiography and Electrocardiography. The Year Book Publishers, Chicago.

Mirowski, M. (1964). Left atrial ectopic rhythm in a patient with rheumatic mitral insufficiency. Proc. Tel-Hashomer Hosp., 3, 119.

- (1966a). Left atrial rhythm. Diagnostic criteria and differentiation from nodal arrhythmias. Amer. $\mathcal{F}$. Cardiol., 17, 203.

- (1966b). Rapid left atrial rhythms. A report of two cases with "dome and dart" $\mathrm{P}$ waves. Israel f. med. Sci., 2, 55.
- (1966c). Electrocardiographic evidence of the existence of ectopic rhythms originating anteriorly in the left atrium. Circulation, 34, 111.

$\longrightarrow$, Neill, C. A., and Taussig, H. B. (1963). Left atrial ectopic rhythm in mirror-image dextrocardia and in normally placed malformed hearts. Report of twelve cases with "dome and dart" $P$ waves. Circulation, 27, 864.

- , and Vure, E. (1966). The occurrence and the mechanism of $P$ wave inversion in lead $I$ in right atrial overloading. Amer. Heart f., 72, 102.

Prinzmetal, M., Corday, E., Brill, I. C., Oblath, R. W., and Kruger, H. E. (1952). The Auricular Arrhythmias, p. 181. Charles C. Thomas, Springfield, Illinois.

Puech, P. (1956). L'activité Électrique Auriculaire Normale et Pathologique. Masson, Paris.

Scherf, D., Blumenfeld, S., and Yildiz, M. (1963). Experimental study of ectopic impulse formation in the left atrium. Cardiologia (Basel), 43, 133.

- , and Cohen, J. (1964). The Atrioventricular Node and Selected Cardiac Arrhythmias. Grune and Stratton, New York. 